\title{
Denoising of Sparse Three- and Four-dimensional Hyperspectral Electron Microscopy Data Using a Total Variational Method
}

\author{
Steven Zeltmann ${ }^{1}$, Andrew Minor ${ }^{1,2}$ and Colin Ophus ${ }^{3}$ \\ ${ }^{1}$ University of California-Berkeley, Berkeley, California, United States, ${ }^{2}$ Lawrence Berkeley National \\ Laboratory, Berkeley, California, United States
}

The advent of direct electron detectors in scanning transmission electron microscopy (STEM) has enabled the acquisition of hyperspectral data such as four dimensional STEM (4D-STEM) and STEM-electron energy loss spectroscopy (STEM-EELS) data at very high scan rates and without the sources of noise that infect data from traditional detectors. The 4D Camera at the Molecular Foundry, for instance, acquires $576^{2}$ pixel diffraction images at 87,000 frames per second [1]. A major limitation of detectors operated at these speeds and the electron counting algorithms used to remove electronic nose is that the total electron dose per image must be very low (perhaps a few thousand electrons per capture). Therefore, in order to make use of the high speed and low noise of high speed counted detectors, it is necessary to apply denoising to the counted data to recover a useful signal.

A wide variety of denoising algorithms exist for image data, many of which have been extended to higher dimensional data. Among the best known is BM3D, which searches for similar "blocks" within the data, then uses correlation between blocks to average the correlated patches and decrease the noise [2]. Recently, machine learning has also been demonstrated to yield effective denoising in higher dimensions [3], though with relatively high computational cost. Here, we extend the total variational (TV) denoising algorithm of Jia and Zhou [4] to three and four dimensions and implement it using OpenMP for parallel execution. Denoising by total variational methods is inherently local, as the variation is computed using local gradients of the signal. The strength of denoising can also be varied for different signal dimensions by controlling the weighting parameters separately for each dimension. This is advantageous for hyperspectral data, where the variation along the scan dimensions should be penalized differently than variation along the detector dimension(s).

Here we present the results of applying total variational denoising to hyperspectral microscopy data, namely 4D-STEM and STEM-EELS mapping. Figure 1 shows the results of total variational denoising applied to a simulated 4D-STEM dataset of a strained, polycrystalline gold foil with $100 \times 80$ scan positions. We include a simply modeled additive background in the data to simulate inelastic scattering and apply Poisson noise to each pattern in the dataset, simulating 1,000 electrons per pattern with a maximum of 1 electron per pixel. This signal level and counting limitation is typical of the fastest counting detectors currently in use [1]. After applying the TV denoising algorithm to the 4D dataset, we recover the diffraction pattern shown in the bottom center. Denoising of this dataset took about 6 minutes on a 4core $3.8 \mathrm{GHz}$ iMac.

TV denoising of STEM-EELS data from a thick nickel nanoparticle is shown in Figure 2. The EELS map shown here was acquired on the TEAM1 microscope, using a Gatan K3 detector and Continuum spectrometer. Each spectrum covers a range of $3 \mathrm{keV}$ and contains approximately 2,000 electrons. Maps of the zero loss peak intensity and the integrated first and second plasmon intensities are shown in Figure 2. Sharp edges are preserved in the plasmon images while the noise within the particle is reduced. The single spectrum and scan average show that substantial smoothing in the individual patterns is achieved while the overall signal closely matches the experimental data. 
TV denoising presents a fast and effective means of recovering signals from the extremely sparse datasets produced by modern electron-counting detectors. The high degree of redundancy in the data permits this remarkable recovery of signals from very weak measurements, as the underlying signal has correlation between scan positions while the noise is uncorrelated. Here, we have shown reconstruction of 4D-STEM and STEM-EELS signals from datasets with signal levels typical of modern detectors operated at full speed. This method is also applicable to chemical mapping by STEM-EDS, where signal is limited by the small collection angle of the detector and high redundancy between neighboring scan locations is also expected. The algorithms used here will be released as an open-source Python package at github.com/sezelt/cyTV4D.

S.E. Zeltmann was supported by STROBE, a NSF Science and Technology Center. Work at the Molecular Foundry was supported by the Office of Science, Office of Basic Energy Sciences, of the U.S. Department of Energy under Contract No. DE-AC02-05CH11231.

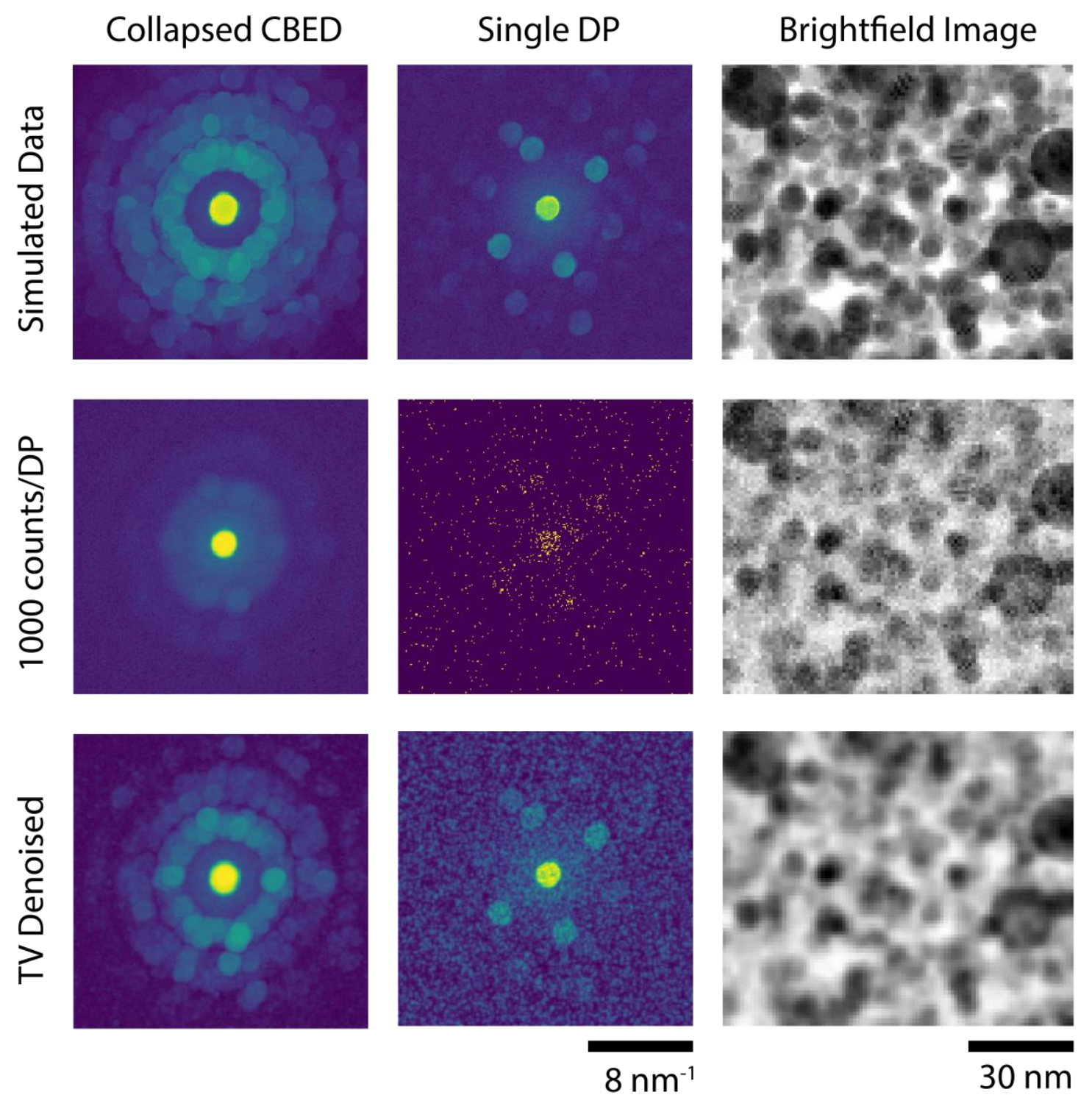


Figure 1. TV denoising of simulated 4D-STEM data. The first column shows an overview of the entire scanning diffraction dataset (the maximum pixel value for the simulation and TV, and the sum for the noisy data since every pixel has a maximum of 1 count). The second column shows a single diffraction pattern from the scan, where the probe sits over two crystallites. The last column shows the virtual brightfield image for the 4D-STEM scan. TV denoising recovers the diffraction disks but real-space features in the brightfield image become blurred.

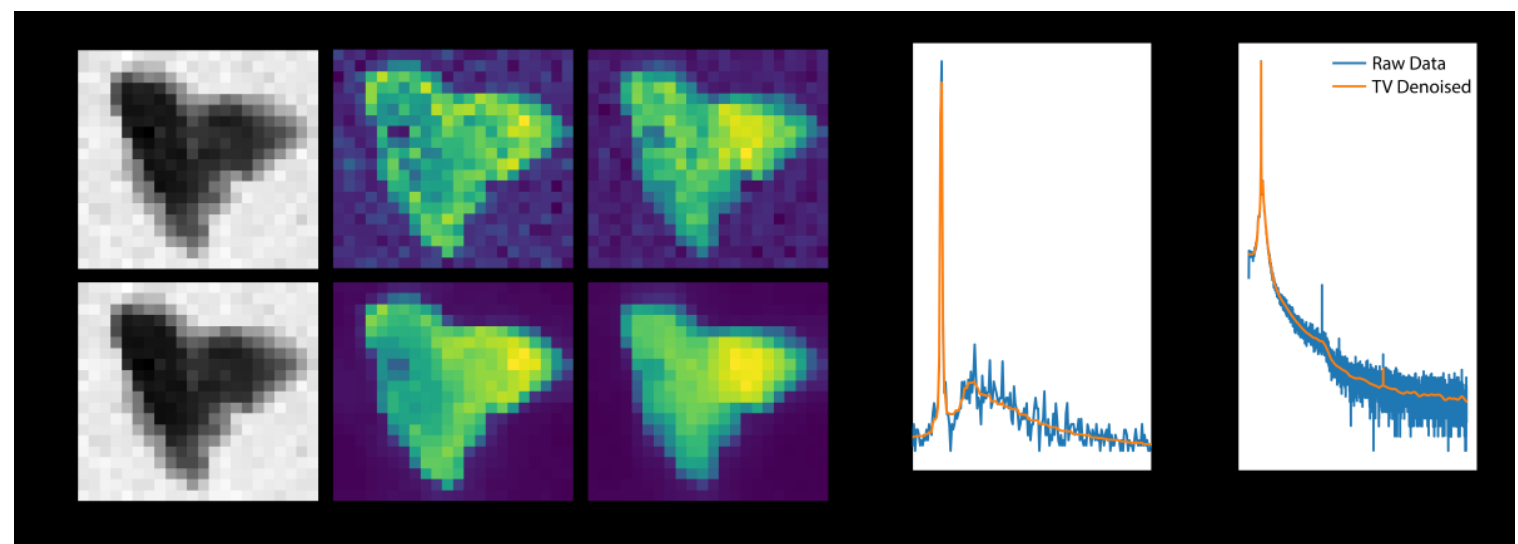

Figure 2. TV denoising of an experimental STEM-EELS map, showing the raw and denoised zero-loss peak image and images of the integrated first and second plasmon peaks for a thick Ni particle. Each EELS spectrum covers a range of approximately $0-3,000 \mathrm{eV}$ and contains about 2,000 electrons.

\section{References}

[1] Ciston et al., Microscopy and Microanalysis (2019) 25(S2): pp.1930-1931

[2] Dabov et al., Proc SPIE (2006) 606414

[3] Batson and Royer, arXiv:1901.11365 [cs.CV]

[4] Jia and Zhou, Avd. Comput. Math (2010) 33:231-241 